\title{
Programa Novotec: Utilização de tecnologias OpenSource para realização do cálculo da distância entre a escola de ensino médio do aluno e os locais com oferta de cursos profissionalizantes presenciais
}

\author{
Felipe S. Silles ${ }^{1}$, Felipe A. F. Kleine ${ }^{1}$, Vagner L. Gava ${ }^{1}$, Celso L. Alves da Silva ${ }^{1}$, \\ Renan Vinicius Ferreira ${ }^{1}$, Kelsey Magalhães Melo ${ }^{1}$, Adriano Galindo Leal ${ }^{1}$ \\ ${ }^{1}$ Instituto de Pesquisas Tecnológicas do Estado de São Paulo, SP, 05508-901, Brazil \\ fsilles@ipt.br, fkleine@ipt.br, vlgavalipt.br, celsosilva@ipt.br \\ rferreira@ipt.br, kelsey@ipt.br, leal@ipt.br
}

\begin{abstract}
This article aims to present the process developed in implementing a solution for technical courses vacancies prioritization based on the geolocation of the teaching institution of a high school student in the public school of São Paulo. The assignment on provides courses by one of the Economic Development Secretariat of the State of São Paulo is carried out through the use of opensource technologies, considering that the choices of the courses were prioritized by proximity of the school. During the registration period that followed the solution implementation, 129,642 new users and approximately 1.5 million page views were found, with the cost absorbed only by the software, without the need to contract third-party services. There where 28,182 assignments, $60 \%$ of which in courses given at institutions less than 5 kilometers from the students' school.
\end{abstract}

Resumo. Esse artigo propõe apresentar o processo desenvolvido na implantação de uma solução para priorização de vagas de cursos técnicos e profissionalizantes gratuitos com base na geolocalização da instituição de ensino do estudante do ensino médio da rede pública estadual de São Paulo. A inscrição em cursos fornecidos pela Secretaria de Desenvolvimento Econômico do Estado de São Paulo é realizada por meio da utilização de tecnologias opensource, considerando-se que as escolhas dos cursos foram priorizadas por proximidade da escola. Durante o período de inscrições que sucedeu a implantação da solução, constatou-se 129.642 novos usuários e aproximadamente 1,5 milhões de visualizações de páginas com o custo absorvido somente pelo software, sem a necessidade de contratação de serviços de terceiros. Foram realizadas 28.182 inscrições, sendo $60 \%$ em cursos ministrados em instituições com menos de 5 quilômetros da escola dos alunos.

\section{Introdução}

A atual demanda por capacitação de jovens alunos da rede de ensino e a introdução ao mercado de trabalho vem mobilizando o governo do estado de São Paulo. Para atender a esta necessidade, o governo projetou soluções de oferecimento de cursos profissionalizantes de cunho técnico para diferentes perfis de alunos presentes na população paulista. Uma destas soluções foi a criação do programa Novotec 
[Secretaria de Desenvolvimento Econômico 2021], que visa a atender a necessidade do mercado de complementação da formação dos estudantes de ensino médio com cursos técnicos e profissionalizantes gratuitos. Estes cursos são oferecidos em ETECs (Escolas Técnicas Estaduais) ou em instituições de ensino públicas do estado de São Paulo.

O estado de São Paulo tem uma área de 248.219,481( $\left.\mathrm{km}^{2}\right)$, e é o décimo segundo em área no Brasil. O programa oferece uma gama com mais de 20 cursos, abrangendo diversas áreas de atuação que os estudantes podem escolher. Como os cursos não são oferecidos em sua totalidade em cada uma das unidades de ensino do estado de São Paulo, torna-se importante o estudo do mapeamento dos locais de oferecimento para que seja possível um melhor atendimento aos alunos.

Segundo o Censo Escolar da Secretaria de Educação do Estado de São Paulo, a grande maioria dos alunos de Ensino Médio pertencem à rede pública estadual. Em 2014, 83,9\% das matrículas do Ensino Médio pertenciam à rede pública estadual, enquanto as $16,1 \%$ restantes dividiam-se entre as unidades federais, municipais e da rede privada [Siqueira 2017]. Desta forma, há uma preocupação com o oferecimento destes cursos técnicos e profissionalizantes na proximidade onde os alunos estudam, evitando deslocamentos exagerados ou mesmo inviáveis dos alunos, aumentando o aproveitamento e diminuindo a possibilidade de evasão. Algumas unidades de ensino por região administrativa do estado de São Paulo podem ser vistas na Figura 1.

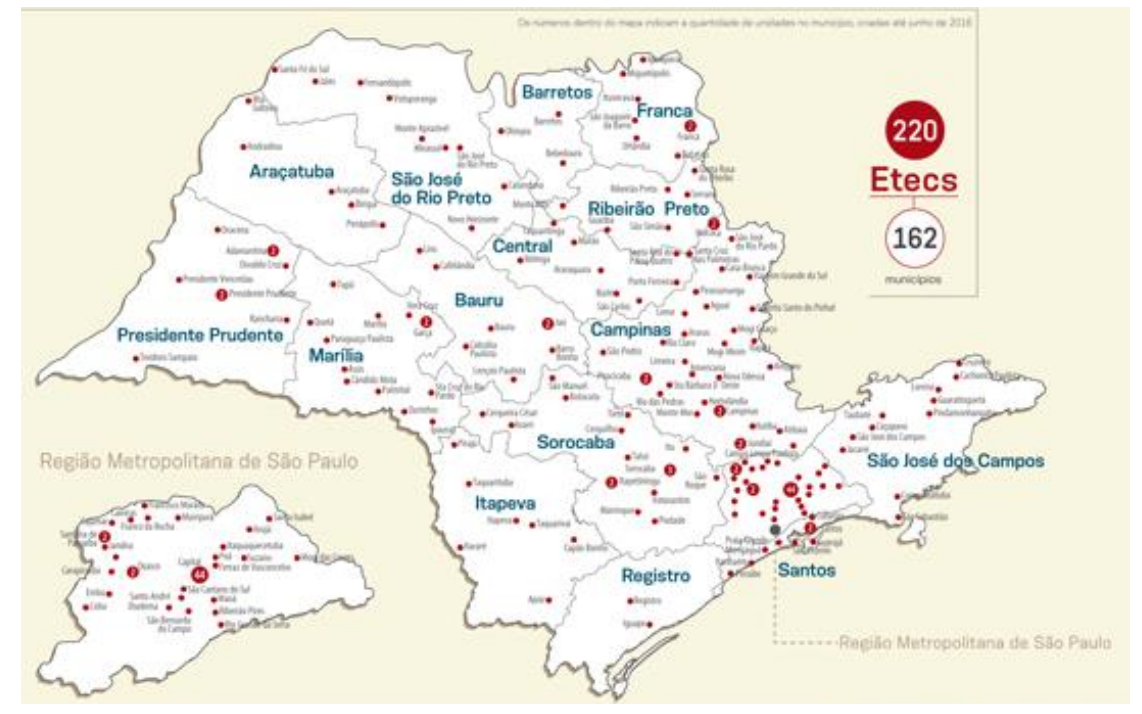

Figura 1. Mapa das ETECs por Região Administrativa (Fonte: SIQUEIRA, 2017)

Com vista no cenário descrito, a Secretaria de Desenvolvimento Econômico do Governo Estado de São Paulo em conjunto com o Instituto de Pesquisas Tecnológicas trabalharam em parceria para propor uma solução adequada ao contexto apresentado para o oferecimento dos cursos técnicos e profissionalizantes.

Para tal, a solução baseou-se na distância entre a unidade de ensino médio dos alunos candidatos aos cursos e as escolas que oferecem esses cursos profissionalizantes por meio de uma solução de baixo custo, utilizando software livre e dos processos, visando a promover os conceitos de governo inteligente [Algebri et al. 2017] por meio da utilização de tecnologias de sistemas de informação geográfica [Tao 2013]. 


\section{Situação/Contexto}

Atualmente a geolocalização está presente em muitos aplicativos que são utilizados no cotidiano. Em uma viagem, por exemplo, pode-se enviar uma determinada localização para o acompanhamento do trajeto, identificação dos locais de interesse próximos da localização atual e outras possíveis aplicações, de modo que, um aplicativo que se propõe utilizar dados geográficos não precisa mais criar o próprio motor de inferência de geolocalização, uma vez que já existem integrações disponíveis criadas por empresas especializadas em fornecer esse tipo de serviço.

Essa integração da geolocalização nos aplicativos é feita pelas chaves de APIs, (Aplication Programing Interface) e os principais provedores atuais são o Mapbox e o Google Maps. Esses serviços facilitam à integração dessa tecnologia nas mais diversas aplicações e as empresas que fornecem esse serviços tem como modelo de negócio a cobrança pelo número de chamadas ao seu serviço disponibilizado, o que encarece a manutenção do sistema, principalmente se o projeto deste aplicativo não contar com recursos alocados para contratação desse serviço e/ou depender da burocracia estatal para sua renovação.

Como alternativa em relação à utilização dos recursos de geolocalização fornecidos por empresas terceiras, esse trabalho tem como objetivo demonstrar uma abordagem com ferramentas opensource utilizadas na criação de um sistema para inscrição de alunos em cursos técnicos de curta duração, indicando a ordenação desses cursos com base no curso selecionado e coordenadas geográficas da escola de ensino médio do aluno.

O processo revelou-se necessário devida à quantidade de vagas dos cursos oferecidos pelo programa Novotec ser limitada, sendo necessário o oferecimento dos cursos nas unidades de ensino técnico profissionalizante mais próximas aos alunos, visando a diminuir as distâncias percorridas, diminuindo a probabilidade de evasão e aumentando o aproveitamento dos cursos oferecidos.

\section{Ações Realizadas}

Esta seção descreve o processo de transformação realizado nos dados brutos para cadastro no banco de dados com extensão geográfica para realização de pesquisas espaciais.

O processo para a preparação dos dados para utilização no sistema pode ser observado na Figura 2. A etapas são detalhadas a seguir.

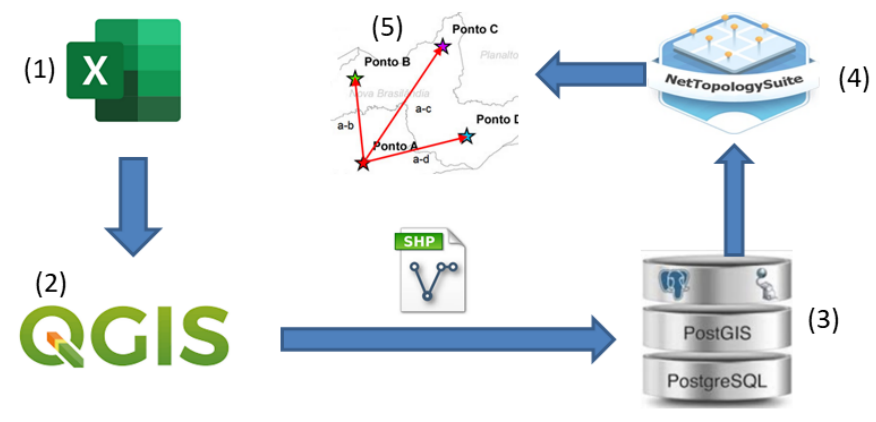

Figura 2. Processo de preparação dos dados geográficos 
A importação dos dados da planilha: de posse dos dados de localização das escolas, foi verificado que parte dessas escolas estavam sem os dados de latitude e longitude preenchidos, portanto nesta etapa foi realizado o preenchimento desses valores faltantes pela conversão de endereço em coordenadas por meio da interface de geocodificação do QGIS [QGIS 2021]. Na Figura 3 é possível observar as informações básicas recebidas de cada escola.

\begin{tabular}{|c|c|c|c|c|c|c|}
\hline & & & & & & \\
\hline 05267000 & 1 CAPITAL & $-23,43794952$ & $-46,7922485111$ & 39111913 & 39166331 & E000059A@EDUCACAO.SP.GOV.BR \\
\hline 05264050 & 1 CAPITAL & $-23,44454956$ & $-46,7851600611$ & 39117266 & $5 \quad 39166325$ & E039342A@EDUCACAO.SP.GOV.BR \\
\hline 05265020 & 1 CAPITAL & $-23,45000076$ & $-46,788700111$ & 39166323 & & E044261A@EDUCACAO.SP.GOV.BR \\
\hline 05267040 & 1 CAPITAL & $-23,43808937$ & $-46,7916603111$ & 39127160 & 39127163 & E457243A@EDUCACAO.SP.GOV.BR \\
\hline 05283010 & 1 CAPITAL & $-23,4353038$ & $-46,775370311$ & 39420878 & 39423113 & E923624A@EDUCACAO.SP.GOV.BR \\
\hline
\end{tabular}

Figura 3. Exemplo dos dados recebidos na planilha

As informações em formato de planilha foram importadas para o software QGIS, aplicativo profissional GIS Livre e de Código Aberto e construído a partir de Software Livre, Free and Open Source Software (FOSS).

Transformação em shapefile: os dados foram importados da planilha para o QGIS e transformados em formato shapefile [ESRI 1998], formato popular de arquivo contendo dados geoespaciais em forma de vetor usado por Sistemas de Informações Geográficas também conhecidos como SIG. Shapefiles espaciais descrevem geometrias: pontos, linhas e polígonos.

Entre outras, essas geometrias podem representar Poços (pontos), Rios (linhas), e Lagos (polígonos), respectivamente. Cada item pode ter atributos que os descrevem (metadados). Na Figura 4 pode ser visto o resultado da transformação da planilha para o formato shapefile.

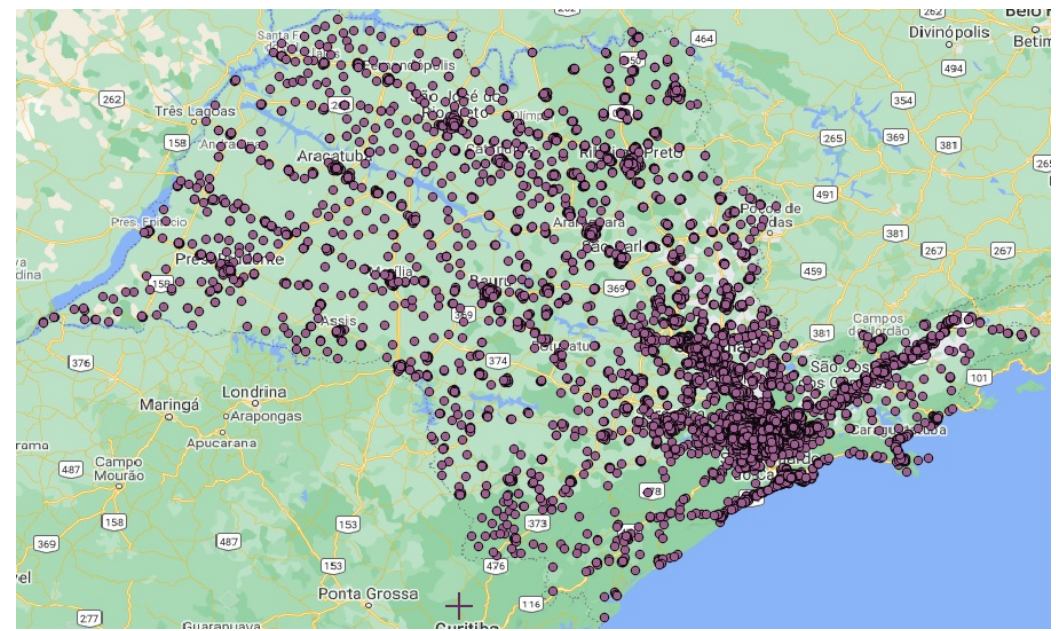

Figura 4. Visualização das escolas no shapefile no Qgis

Conversão do shapefile para tabela no PostgreSQL com a extensão PostGIS: com o arquivo shapefile gerado no QGIS, foi realizada a importação dos dados, utilizando um aplicativo executável Shp2pgSQL [QGIS Project 2021] que permite a conversão de 
arquivos shapefiles em arquivos escritos em SQL que podem ser importados diretamente no pgAdmin, uma ferramenta de administração do PostgreSQL [PGAdmin 2021].

Para trabalhar com dados geográficos no PostgreSQL, foi realizada a instalação do PostGIS [PostGis 2021], uma extensão para o sistema de banco de dados objeto-relacional PostgreSQL [PostgreSQL 2021] e que permite que objetos SIG (Sistema de Informação Geográfica) sejam armazenados em banco de dados. O PostGIS inclui suporte a índices espaciais baseado em GiST R-Tree, e funções para análise e processamento de objetos SIG.

Na Figura 5 é possível verificar o resultado da importação para a tabela do postgres.

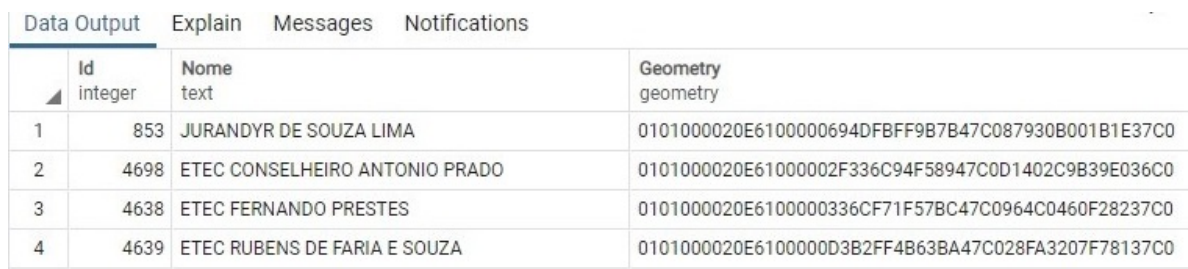

Figura 5. Resultado da importação para PostgreSQL com Postgis

Configuração da extensão NetTopologySuite para utilização dos tipos geográficos e realização do cálculo de distâncias na programação do C\#: após a importação dos dados para a tabela do banco de dados foi realizada a configuração do NTS Net Topology Suite [NTS 2021], uma API para modelar e manipular geometria linear bidimensional, fornecendo vários predicados geométricos e funções para facilitar a manipulação dos dados geográficos.

Apresentação dos cursos pela menor distância da escola onde o aluno está matriculado: na Figura 6 é possível observar o resultado das escolas de ensino técnico mais próximas da escola de ensino médio que o aluno estuda que oferecem o curso escolhido no momento da inscrição. Ressalta-se que a escolha da escola de ensino médio como referência foi realizada, pois pressupõe-se que o aluno se encaminhe da escola de ensino médio para fazer o curso técnico em retorne do curso técnico para a escola de ensino médio.

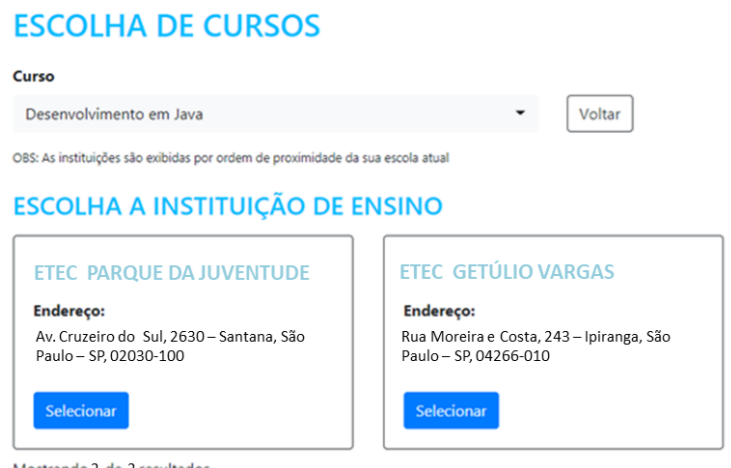

Figura 6. Exemplo de apresentação das turmas do curso mais próximo da escola do aluno 


\section{Resultados}

Durante o período de inscrições, que aconteceu de 03 de junho até o dia 17 de junho de 2019, foi registrada a abertura de 198.573 sessões no site, dados que podem ser observados na Figura 7.

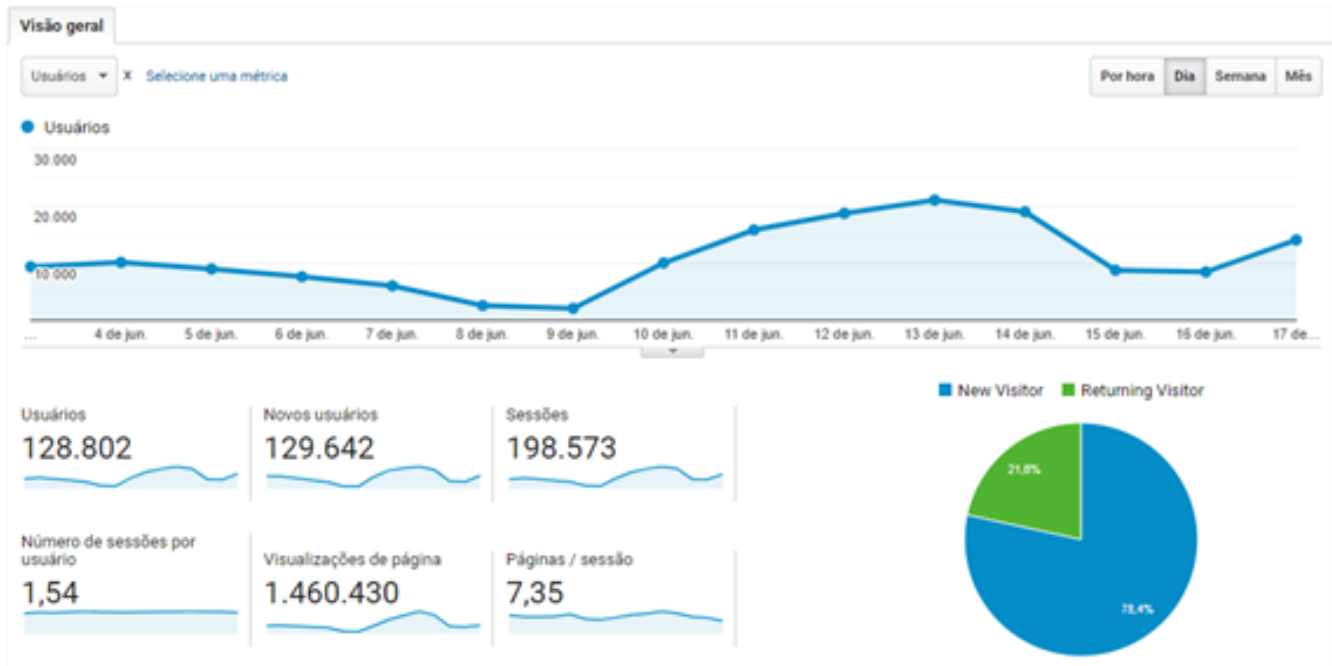

Figura 7. Dados de sessão dos usuários extraídos do Google Analytics

Uma sessão é o período de tempo que um usuário está interagindo com o site, totalizando 129.642 novos usuários e aproximadamente 1,5 milhões de visualizações de páginas. Com o número de sessões registradas no período de inscrição é possível estimar a quantidade de chamadas de API para cálculo de distância que seria necessário com a utilização de um serviço comercial. Na Figura 8 é possível verificar uma estimativa de custo em dólar com o serviço do google maps.

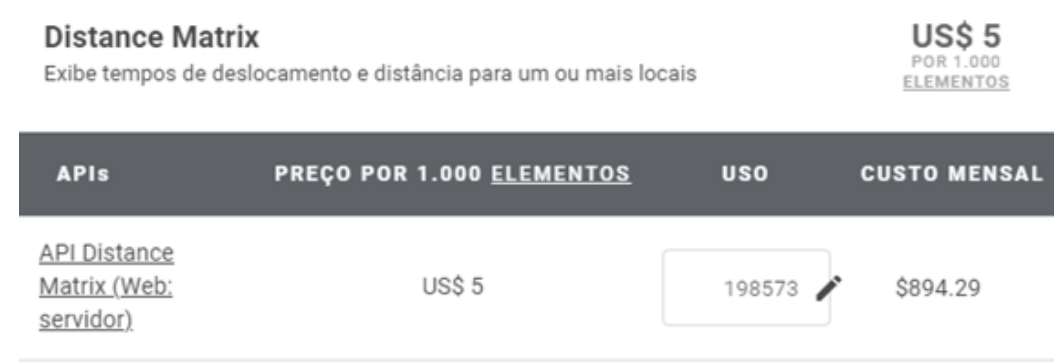

Figura 8. Estimativa de custo com requisições a API do Google Maps

Com a utilização das ferramentas opensource descritas na Figura 2, esse custo pode ser absorvido pelo software sem a necessidade de contratação de API de terceiros, viabilizando a entrega do sistema e utilizando as funcionalidades de geolocalização para facilitar a apresentação das escolas técnicas mais próximas que ofereciam os cursos de interesse do aluno.

Ao final do processo, com 28.182 inscrições, foi realizada a contabilização por meio de consulta ao banco de dados das distâncias entre a escola onde o aluno estuda e a escola escolhida pelo aluno para fazer o curso. 
Na Figura 9 é possível observar que a maioria dos alunos escolheram cursos com distância menor que dois quilômetros do seu local de estudo.

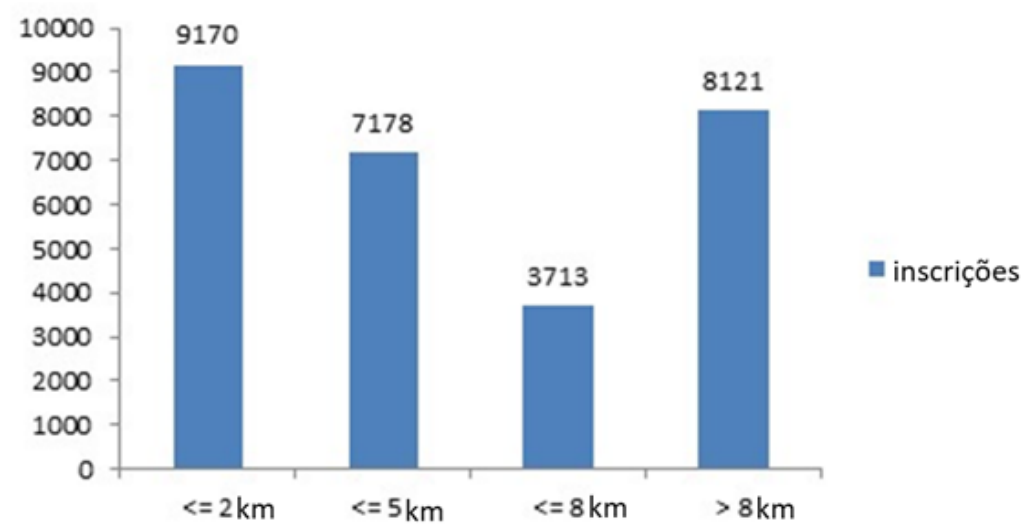

Figura 9. Quantidade de alunos inscritos pela distância entre as escolas

Como a escolha do curso foi livre e o objetivo foi ajudar o aluno a escolher cursos próximos à escola onde cursava ensino médio, foi possível identificar que as inscrições com distância menor que 5 quilômetros atingiram aproximadamente $60 \%$ do total de inscrições realizadas, diminuindo a possibilidade da escolha de cursos de interesse mais distantes do local de moradia do aluno.

É importante ressaltar que o projeto descrito neste artigo teve como objetivo acompanhar a realização das inscrições até matrícula dos alunos, sem o acompanhamento da efetiva realização das disciplinas, executada por outros sistemas do Centro Paula Souza da Secretaria de Desenvolvimento Econômico do Estado de São Paulo.

\section{Lições Aprendidas}

O desenvolvimento de tecnologias opensource e o aumento da utilização dessas tecnologias são uma oportunidade para utilização em aplicações de governo visando a diminuição de custos e mantendo alta a qualidade do produto desenvolvido.

A escolha das ferramentas explicitadas na Figura 2 mostrou-se eficaz na resolução do problema de apresentação dos cursos técnicos profissionalizantes mais próximos aos alunos, que comparada à utilização de serviços de terceiros, apresentaria um custo aproximado de 900 dólares para o período da inscrição.

Considerando-se ainda que o programa tem como objetivo a realização das inscrições duas vezes ao ano e que a tendência é de aumento da procura pelos alunos, esses gastos poderiam ser estimados em torno de 1800 dólares por ano. Além do valor que deixou de ser pago, note-se que o maior problema é que nem sempre será possível garantir a renovação do contrato desse serviço, em função de eventuais dificuldades orçamentárias e burocráticas dos órgãos públicos envolvidos.

A utilização de técnicas de sistemas de informações geográficas empregadas, não somente para análise de dados posterior aos fatos, mas também no momento que o sistema está em contato com o usuário final, pode ser útil como suporte no emprego de políticas que visem ao melhor aproveitamento dos recursos públicos, nesse caso, na oferta de vagas 
em cursos de curta duração, pois acredita-se que o aluno que escolhe fazer o curso técnico profissionalizante mais próximo da escola de ensino médio onde estuda tenha maior facilidade para se locomover até o local do curso, o que pode contribuir para o aumento dos índices de conclusão.

Por fim, como trabalho futuro, sugere-se o acompanhamento dos índices de conclusão para serem utilizados para avaliar se os alunos que escolheram cursos próximos de onde estão estudando tem um maior índice de conclusão dos cursos ofertados como forma de aumentar a abrangência desse programa.

\section{Agradecimentos}

Os autores desejam agradecer ao suporte recebido através dos processos $n^{\circ}$ \#2019/01664-6 e \#2017/50343-2, Fundação de Amparo à Pesquisa do Estado de São Paulo (FAPESP).

\section{Referências}

Algebri, H. K., Husin, Z., Abdulhussin, A. M., and Yaakob, N. (2017). Why move toward the smart government. In 2017 International Symposium on Computer Science and Intelligent Controls (ISCSIC), pages 167-171. IEEE.

ESRI (1998). ESRI shapefile technical description. Acesso em 20 de abril de 2021, disponível em https://www.esri.com/content/dam/esrisites/ sitecore-archive/Files/Pdfs/library/whitepapers/pdfs/ shapefile.pdf.

NTS (c2021). Software para manipulação de dados geográfico. Acesso em 20 de abril de 2021, disponível em https:// nettopologysuite.github.io/.

PGAdmin (c2021). Software administrado PGAdmin. Acesso em 20 de abril de 2021, disponível em https: / / www.pgadmin.org/.

PostGis (c2021). Extensão espacial PostGis. Acesso em 20 de abril de 2021, disponível em https://postgis.net/.

PostgreSQL (c2021). Sistema gerenciador de banco de dados postgresql. Acesso em 20 de abril de 2021, disponível em https: / / www . postgresql org/.

QGIS (c2021). Software QGIS. Acesso em 20 de abril de 2021, disponível em https : //qgis.org/pt_BR/site/.

QGIS Project (2021). Software conversor shp2pgsql. Acesso em 20 de abril de 2021, disponível em https://docs.qgis.org/3.16/en/docs/training_ manual/spatial_databases/import_export.html?highlight= shp2pgsql.

Secretaria de Desenvolvimento Econômico, G. d. E. d. S. P. (c2021). Novotec. Acesso em 26 de março de 2021, disponível em http: / / www . novotec.sp.gov . br.

Siqueira, T. M. A. d. (2017). A rede de educação pública do estado de são paulo: uma análise comparativa entre as escolas técnicas do estado de são paulo (etecs) e as demais escolas regulares.

Tao, W. (2013). Interdisciplinary urban gis for smart cities: advancements and opportunities. Geo-spatial Information Science. 